\title{
BMJ Open Motor control exercise for symptomatic lumbar disc herniation: protocol for a systematic review and meta-analysis
}

\author{
Mohammad Reza Pourahmadi, ${ }^{1}$ Morteza Taghipour, ${ }^{2}$ Ismail Ebrahimi Takamjani, ${ }^{1}$ \\ Mohammad Ali Sanjari, ${ }^{3}$ Mohammad Ali Mohseni-Bandpei, ${ }^{4,5}$ Abbas Ali Keshtkar ${ }^{6}$
}

To cite: Pourahmadi MR, Taghipour M, Ebrahimi Takamjani I, et al. Motor control exercise for symptomatic lumbar disc herniation: protocol for a systematic review and metaanalysis. BMJ Open 2016;6: e012426. doi:10.1136/ bmjopen-2016-012426

- Prepublication history and additional material is available. To view please visit the journal (http://dx.doi.org/ 10.1136/bmjopen-2016012426).

Received 25 April 2016 Revised 30 August 2016 Accepted 1 September 2016

CrossMark

For numbered affiliations see end of article.

Correspondence to Morteza Taghipour; taghipour-morteza@hotmail. com

\section{ABSTRACT}

Introduction: Lumbar disc herniation (LDH) is a common condition in adults and can impose a heavy burden on both the individual and society. It is defined as displacement of disc components beyond the intervertebral disc space. Various conservative treatments have been recommended for the treatment of $\mathrm{LDH}$ and physical therapy plays a major role in the management of patients. Therapeutic exercise is effective for relieving pain and improving function in individuals with symptomatic LDH. The aim of this systematic review is to evaluate the effectiveness of motor control exercise (MCE) for symptomatic LDH.

Methods and analysis: We will include all clinical trial studies with a concurrent control group which evaluated the effect of MCEs in patients with symptomatic LDH. We will search PubMed, SCOPUS, PEDro, SPORTDiscus, CINAHL, CENTRAL and EMBASE with no restriction of language. Primary outcomes of this systematic review are pain intensity and functional disability and secondary outcomes are functional tests, muscle thickness, quality of life, return to work, muscle endurance and adverse events. Study selection and data extraction will be performed by two independent reviewers. The assessment of risk of bias will be implemented using the PEDro scale. Publication bias will be assessed by funnel plots, Begg's and Egger's tests. Heterogeneity will be evaluated using the $\mathrm{I}^{2}$ statistic and the $\chi^{2}$ test. In addition, subgroup analyses will be conducted for population and the secondary outcomes. All meta-analyses will be performed using Stata V.12 software.

Ethics and dissemination: No ethical concerns are predicted. The systematic review findings will be published in a peer-reviewed journal and will also be presented at national/international academic and clinical conferences.

Trial registration number: CRD42016038166.

\section{INTRODUCTION}

Lumbar disc herniation (LDH) and disc protrusion are one of the most common spinal degenerative disorders, which can lead to low back pain (LBP) and radicular leg pain. ${ }^{1}$ It is a pathological condition that frequently

\section{Strengths and limitations of this study}

This systematic review and meta-analysis wil evaluate the effectiveness of motor control exercise for symptomatic lumbar disc herniation. There will be no limitation by language and grey literature will be included if identified through the searches.

- This protocol was prepared according to the Preferred Reporting Items for Systematic Review and Meta-Analysis Protocols (PRISMA-P) recommendations.

- Full-text screening, data extraction and risk of bias assessment of included primary studies will be conducted by two reviewers independently.

- Some relevant unpublished trials with negative findings that meet our inclusion criteria might be missed (publication bias). Therefore, funnel plots will be used to detect possible publication bias in order to reach an objective conclusion.

affects the spine in young and middle-aged adults. $^{2}$ This condition is defined as a displacement of disc components (nucleus pulposus or annulus fibrosis) beyond the

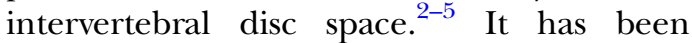
shown that $\mathrm{LDH}$ is the most common cause of radiculopathy $(90 \%))^{6}{ }^{7}$ The highest prevalence of disc herniation is among adults aged 30-50 years, with a male to female ratio of 2:1. ${ }^{4}$ In adults aged 2555 years, about $95 \%$ of $\mathrm{LDH}$ predominantly occurs at the L4-5 and L5-S1 spinal levels. ${ }^{4}$ Disc herniation above these levels is more common in adults aged over 55 years. ${ }^{4}$ However, it has been reported that $\mathrm{LDH}$ is not always accompanied by clinical symptoms such as LBP (asymptomatic LDH). ${ }^{1}$ Studies by Boden $e t a l^{8}$ and Jensen et a ${ }^{9}$ indicated that $24-27 \%$ of asymptomatic subjects have disc herniation. In addition to the general wear and tear (degenerative joint disease) that comes with ageing, many risk factors have been reported for $\mathrm{LDH}$, such as gender, weight, profession, smoking, psychosocial 
factors, exposure to vibration and sedentary lifestyle. ${ }^{10-12}$ The cardinal symptoms of symptomatic LDH include LBP, radicular leg pain, sensory loss (paraesthesia, numbness and tingling), muscle weakness and incontinence (rare) ${ }^{13}$

A variety of therapeutic interventions have been proposed for the treatment of symptomatic LDH, including non-invasive treatments, minimally invasive procedures and surgery. ${ }^{14}$ It was demonstrated that symptomatic LDH could be effectively treated both surgically and non-surgically. ${ }^{15}$ Non-invasive (non-surgical) treatments are considered to be a first-line choice for most cases. ${ }^{16}$ Bed rest, physical therapy, comfortable positioning, manipulation and drug therapy (non-steroidal antiinflammatory drugs) are the most used non-invasive treatments. $^{17}$

Physical therapy programmes are often recommended for the treatment of pain and restoration of functional and neurological deficits associated with symptomatic $\mathrm{LDH}^{18}$ Active exercise therapy, which is a type of physical therapy programme, is usually preferred to passive modalities. ${ }^{19}$ There are a number of exercise programmes for the treatment of symptomatic LDH, such as activity as usual, aerobic activity (eg, walking, cycling), directional preference (McKenzie approach), flexibility exercises (eg, yoga and stretching), proprioception/ coordination/balance (medicine ball and wobble/tilt board), motor control and strengthening exercises. ${ }^{20}$ Motor control exercises (MCEs) or stabilisation or core stability exercises are a common type of therapeutic exercise prescribed for patients with symptomatic LDH. MCEs are designed to re-educate the co-activation pattern of abdominals, paraspinals, gluteals, pelvic floor musculature and diaphragm. ${ }^{21} 22$ The biological rationale for MCEs is primarily based on the idea that the stability and control of the spine are altered in patients with LBP. ${ }^{23}{ }^{24}$ A MCE programme begins with recognition of the natural position of the spine (mid-range between lumbar flexion and extension range of motion), considered to be the position of balance and power for improving performance in various sports. ${ }^{25}$ Initial low-level sustained isometric contraction of trunk-stabilising musculature and their progressive integration into functional tasks is the requirement of MCEs. ${ }^{22}{ }^{26}$ MCE is usually delivered in 1:1 supervised treatment sessions and sometimes includes palpation, ultrasound imaging and/or the use of pressure biofeedback units to provide feedback on the activation of trunk musculature. ${ }^{27}$

\section{How the intervention might work}

Control of the spine is complex and depends on wellcoordinated, deep-trunk musculature. The deep-trunk musculature, which originates and inserts segmentally on lumbar vertebrae, can maintain the stiffness of the spine by controlling intersegmental motion and spinal curvature. ${ }^{28}$ Hodges and Richardson ${ }^{29}{ }^{30}$ indicated that patients with LBP may have a delayed activity onset of the deep-trunk musculature in dynamic tasks that challenge control of the spine. Furthermore, a reduction of the cross-sectional area (CSA) of the multifidus muscle on the affected segment has been found in patients with symptomatic LDH. ${ }^{31}{ }^{32}$ Fortin et $a \hat{l}^{31}$ also reported that patients with symptomatic LDH have a smaller erector spinae functional CSA (FCSA) (lean muscle mass) and FCSA/CSA ratio on the side of herniation. Position sense of the extensor musculature of the trunk has been shown to be altered in patients with $\mathrm{LBP}^{33}$ The MCE approach uses motor learning principles to facilitate coordination of the deep-trunk musculature of the spine. It seems that a MCE can alleviate pain, improve functional capacity, restore motor control, enhance the size of the CSA and strengthen trunk, abdominal and paraspinal musculature.

\section{Literature review}

To date, several systematic reviews have looked at the effects of conservative management in patients with LDH. A systematic review and meta-analysis by Hahne et at in 2010, which included 18 separate trials up to 2008 , concluded that conservative management is better than no treatment in reducing pain and disability. The study consisted of English-language articles only. ${ }^{6}$ In another study, Jacobs $e t a l^{34}$ compared the effectiveness of surgery with conservative management for LDH. Five studies were included up to October 2009. The findings of the study showed that early surgery in patients with LDH results in better short-term leg pain relief than prolonged conservative management, but no significant differences were found between surgery and usual conservative management in any of the clinical outcomes after 1- and 2-years' follow-up. ${ }^{34}$ However, the studies included were of low quality and a definite conclusion could not be reached owing to their high heterogeneity.

\section{Why it is important to do this systematic review}

Since the number of patients with LDH has increased over the past few decades, ${ }^{35}$ and because systematic reviews on this topic are out of date, a new systematic review of the literature is needed. The latest available systematic review and meta-analysis comparing the effectiveness of surgery with conservative treatments for $\mathrm{LDH}$ was based on databases up to October 2009. ${ }^{34}$ However, it did not compare conservative treatments with each other in adult patients with symptomatic LDH. As mentioned earlier, active exercise therapy can provide better outcomes than passive modalities. ${ }^{19}$ MCEs are a popular approach for clinicians and researchers in the treatment of patients with LDH and many studies have been published on this topic. ${ }^{36-38}$ To the best of our knowledge, this systematic review and meta-analysis will evaluate, for the first time, the effectiveness of MCEs for symptomatic $\mathrm{LDH}$. Furthermore, no restriction of the language of publication will be applied in this review. The authors believe that a well-conducted systematic review and 
meta-analysis is important to better inform clinicians, therapists and patients about the effectiveness of MCEs.

\section{Objectives}

The objective of this investigation is to systematically review the literature to determine the effectiveness of MCEs in patients with symptomatic LDH.

\section{METHODS}

The methods adopted for this review are compliant with the recommended PRISMA (Preferred Reporting Items for Systematic Review and Meta-Analysis) checklist guidelines for systematic reviews. In addition, the PRISMA flow diagram ${ }^{39}$ will be used to describe the number of primary studies that are included and excluded in each stage of the selection process (figure 1). This protocol has been prepared with regard to the PRISMA-P 2015 guidelines and has been registered in the international prospective register of systematic reviews (PROSPERO; Registration No CRD42016038166; http://www.crd.york. ac.uk/PROSPERO).

\section{Selection criteria}

Study type

Studies will be screened for selection according to the review objectives and Participants, Interventions, Comparisons, Outcomes (PICO) criteria. We will include all clinical trials with concurrent control groups in this systematic review and meta-analysis. Studies assessing review articles, proceedings, case studies and case reports will be excluded. No restrictions to the language of publication will be applied in the selection of the primary studies. Non-English articles will be translated appropriately by free language translators on the web (Google translate, Bing, ImTranslator, Babelfish and AppliedLanguages) to assess their inclusion.

\section{Population}

The population will comprise non-, pre- and postsurgery groups of adult patients with symptomatic LDH. Studies will be included if they involve adult patients ( $\geq 18$ years) of both genders with referred leg symptoms, with or without LBP, where at least $75 \%$ of the participants have symptomatic LDH confirmed by MRI or CT. ${ }^{6}$ Studies confirming or justifying LDH only with myelography will be excluded since disc herniations are not directly visualised by this technique. ${ }^{40}$ The term 'herniation' is defined as displacement of the nucleus and/or annulus fibrosus through a tear of the annulus fibrosus. ${ }^{41}$ It includes synonymous terms such as prolapse, protrusion and sequestration, but the term disc bulging is not sufficient. Studies with specific pathology, such as systemic inflammatory diseases, malformations, fractures, spondylolisthesis, scoliosis, infections, tumours and osteoporosis, will be excluded.

\section{Interventions}

A MCE programme could be described as facilitation of the deep musculature of the spine (primarily the transversus abdominis or multifidus) at low-level sustained isometric contraction, integrated into exercise and finally, progressing into functional tasks. ${ }^{22} 2642$ A MCE programme improves the ability to ensure stability of the neutral spine position. ${ }^{21}$ We will consider studies in which MCE is described as motor control, core stability or a specific stabilisation exercise and/or the study describes exercise aiming to facilitate, activate, restore, train or improve the function of the deep musculature of the spine. ${ }^{27} 43$ All these different names of MCEs will be used in our search syntax. In addition, we will include trials evaluating Pilates, because the principles of Pilates may overlap with the principles of a motor control intervention. ${ }^{44}$ It is important that all the studies included focus on specific muscle activity in their training programme and if a trial consists of specific stabilisation without consideration of specific muscle activity it will be excluded. ${ }^{43}$ Furthermore, when MCEs are used in addition to other treatments in primary studies, they need to represent at least $50 \%$ of the total treatment programme to be included. ${ }^{27}$

\section{Comparators}

General therapeutic exercises or any other physical therapy intervention, surgery and placebo/sham or control group.

\section{Outcomes}

The primary outcomes of interest will be pain intensity and functional disability. The secondary outcomes are as follows: functional tests, muscle thickness, quality of life, return to work, muscle endurance and adverse events.

\section{SEARCH METHODS FOR IDENTIFICATION OF STUDIES Electronic searches}

The first author (MRP) will perform electronic searches in the following databases between 1 January 1990 and 31 April 2016.

- PubMed/Medline (NLM)

- SCOPUS

- Physiotherapy Evidence Database (PEDro)

- SPORTDiscus (EBSCO)

- Cumulative Index to Nursing and Allied Health Literature (CINAHL)

- The Cochrane Central Register of Controlled Trials (CENTRAL)

- EMBASE

- ClinicalTrials.gov

\section{PubMed search strategy}

The details of the PubMed database search syntax are presented in online supplementary appendix 1 . The syntax of this systematic review is a combination of $\mathrm{MeSH}$ terms and free text words. The syntax will be 
Figure 1 The screening process of the study.

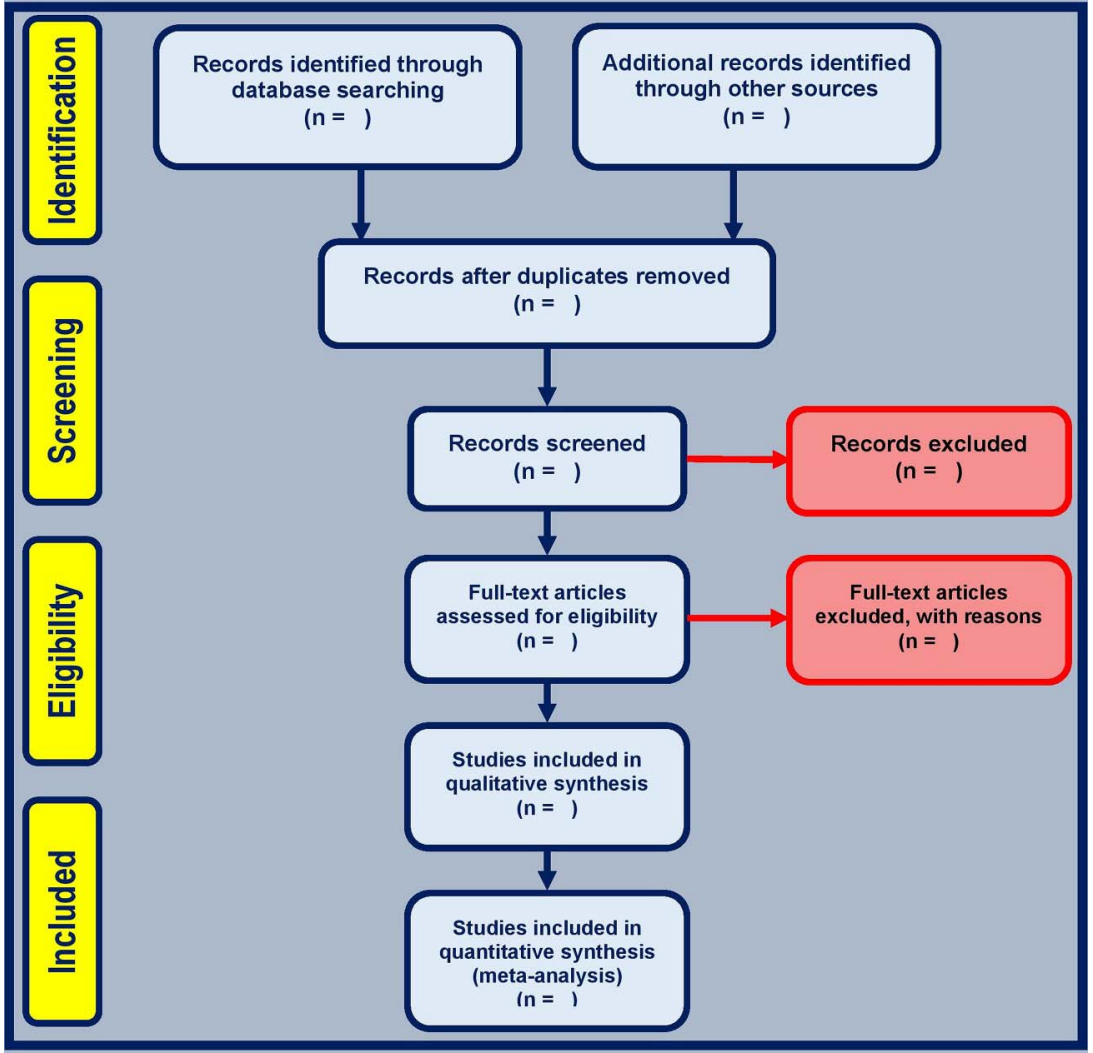

adopted for other databases. We will use PubMed's 'My NCBI' (National Center for Biotechnology Information) email alert service for identification of newly published systematic reviews using a basic search strategy.

If we identify additional relevant keywords during any of the electronic or other searches, we will modify the electronic search strategies to incorporate these terms and document the changes. During searching the electronic databases or reviewing reference lists in identified studies, no restrictions on the language of publication will be imposed.

\section{Searching other resources}

Manual searches will include scanning of reference lists of included studies and similar reviews, journals that publish the most relevant research articles or reviews and other grey literature for relevant references.

Grey literature refers to reports that are difficult to locate via conventional channels and include reports, theses or dissertations, conference proceedings, newspapers, websites, fact sheets, policy documents and unpublished research and data. ${ }^{45} 46$ These documents are not considered to be formally published in academic sources (ie, books or journals). ${ }^{45}$ The Institute of Medicine Standards for Systematic Review and the Cochrane Handbook for Systematic Reviews of Interventions suggest incorporating grey literature in systematic reviews. ${ }^{46} 47$

If we find research that seems to match our objectives, we will contact the corresponding author(s) for missing information and enquire about the existence of further trials. We will use recommended strategies for electronic surveys to enhance response rates. ${ }^{48}$ Specifically, we will (i) send an email to the corresponding author(s), explain the review objectives and request his/her/their data; and (ii) send reminder emails at 2-, 7-, 10- and 14-week intervals after the first email. We will inform all authors that their research will be appropriately cited and they will be acknowledged in our article. If we do not receive a response from the corresponding author (s) after five emails, we will exclude the research.

\section{Data collection and analysis \\ Selection of studies}

First, two reviewers (MRP, MT) will scrutinise titles and abstracts of all primary articles that meet the search strategy in order to determine studies eligible for inclusion. Then, the same two reviewers will independently evaluate the full text of potentially relevant non-duplicated articles. Conflicts will be resolved by discussion to reach consensus. When consensus is not reached, a third reviewer (MAS) will act as an arbitrator. Agreement between the two reviewers will be evaluated and reported using $\kappa$ statistics and overall agreement.

\section{Data extraction}

Data extraction from primary articles will be performed independently by two reviewers (MRP, MT), using a quantitative data extraction form. The data extraction form for clinical trials with concurrent control groups 
will be piloted previously. Any discrepancies will be resolved by consensus between the two reviewers and, when this is not possible, a third reviewer (MAS) will act as an arbitrator and make a decision on the data entered.

The following data will be extracted from all the included studies:

1. Study characteristics: first author's name, year of publication, country in which the study was performed, study design, size of the sample and duration of follow-up.

2. Participants' characteristics: age, gender, number, pre-/ non- or postsurgery population and ethnicity.

3. Intervention and comparator details: sample size for each treatment group, blinding, type, surgery, frequency and duration of the exercise programmes, withdrawals and dropouts.

4. Outcome measures: pain intensity, scales and questionnaires used to assess pain, total score of functional disability, disability questionnaires, type of functional tests and their methods of assessment, instrument for measuring muscle thickness, instruments setting parameters, name of muscles and muscles' thickness (size), differences in changes of muscles thickness, indicators of return to work (eg, time to partial and to full return to work), return to work at different times, tests used to assess muscle endurance, scales and questionnaires used to measure quality of life.

\section{Dealing with missing data}

We will try to contact the corresponding authors of studies by email, if it is necessary to obtain data missing from published articles. However, if the authors do not respond to queries, we will calculate the missing data from other measures or estimate them from the most similar study.

\section{Assessment of risk of bias of included studies}

Assessment of the risk of bias (RoB) and methodological quality will be implemented by two reviewers (MRP, MT) independently considering the items according to the PEDro scale. ${ }^{49}$ The PEDro scale is based on the Delphi list and has been developed by Verhagen et $a l^{50}$ Maher et $a l^{49}$ indicated that the reliability of the total PEDro score, based on consensus judgements, is acceptable. Therefore, they concluded that the scale can be used in systematic reviews of physical therapy clinical trials with control groups. ${ }^{49}$ The PEDro scale items are illustrated in figure 2. Items 2-9 relate to the internal validity of an article, items 10 and 11 provide sufficient statistical information to enable appropriate interpretation of the results. Item 1 refers to the external validity (or 'generalisability' or 'applicability' of the trial) and thus is not included in the total PEDro score. ${ }^{51} 52$ In addition, primary studies which attain scores of $\geq 6$ on the PEDro scale are considered 'high quality'. Studies with a PEDro score of 4 or 5 are considered 'fair quality' and those with scores of $\leq 3$ are considered 'poor quality'. The
PEDro scale has been used in other systematic reviews. ${ }^{52-54}$

\section{Assessment of heterogeneity}

All analyses will be performed using Stata V.12 software (StataCorp LP, College Station, Texas, USA) on a personal laptop. Heterogeneity among primary studies will be evaluated by the $\mathrm{I}^{2}$ statistic and $\chi^{2}$ test as recommended by the Cochrane Handbook for Systematic Reviews of Interventions. ${ }^{46}$ We will interpret the $\mathrm{I}^{2}$ statistic using the following guide: ${ }^{55}$

- $0-40 \%=$ no important heterogeneity;

- 30-60\%=moderate heterogeneity;

- 50-90\%=substantial heterogeneity;

- 75-100\%=considerable heterogeneity.

We will consider heterogeneity before conducting pooled analysis. When substantial heterogeneity $\left(\mathrm{I}^{2}>50 \%\right)$ is evident among the studies, the results will be presented in the text qualitatively and we will not pool them. The decision to use the random-effects model will be based on our understanding of whether or not all included trials share a common effect size and not only on results of tests for statistical heterogeneity. ${ }^{56}$ Therefore, when $\mathrm{I}^{2}$ values are slightly higher than $50 \%$ and there is overlap between the CIs in visual inspection of the forest plot, we will combine the results into a meta-analysis using a random-effects model. ${ }^{27}{ }^{43}$ A value of $\mathrm{p}<0.05$ will be considered as statistically significant.

\section{Assessment of publication bias}

When a sufficient number of studies $(\geq 10)$ are identified, publication bias will be explored by a funnel plot (ie, plots of study results against precision) and Begg's and Egger's tests. We will not assess publication bias when $<10$ studies are available for analysis since the tests for publication bias yields unreliable results.

\section{Data synthesis}

\section{Descriptive analysis}

All included primary studies will be read in detail and presented in two separate tables. The first table will provide details of the assessment of RoB among included studies. The second table will consist of study characteristics, patient characteristics, sample size, duration of complaint, intervention and setting, and outcome data/results. Pain intensity, functional disability, functional tests, muscle thickness, return to work, muscle endurance and adverse events mean scores, together with their range $(\mathrm{SD} / 95 \% \mathrm{CI})$ will be reported. We expect that there will be a sufficient number of clinical trial studies to carry out a meta-analysis for the primary and secondary outcomes; however, the decision to complete a meta-analysis will depend on the number of these types of study identified in the review. To perform a meta-analysis, a minimum of two studies will be required. ${ }^{57}$ Stata V.12 software will be used for meta-analysis. Dichotomous data will be analysed using the risk ratio measure with its $95 \%$ CIs, whereas 
PEDro Scale

1. Eligibility criteria were specified

2. Subjects were randomly allocated to groups (in a crossover study, subjects were randomly allocated an order in which treatments were received)

3. Allocation was concealed

4. The groups were similar at baseline regarding the most important prognostic indicators

5. There was blinding of all subjects

6. There was blinding of all therapists who administered the therapy

7. There was blinding of all assessors who measured at least one key outcome

8. Measures of at least one key outcome were obtained from more than $85 \%$ of the subjects initially allocated to groups

9. All subjects for whom outcome measures were available received the treatment or control condition as allocated or, where this was not the case, data for at least one key outcome was analysed by "intention to treat"

10. The results of between-group statistical comparisons are reported for at least one key outcome

11. The study provides both point measures and measures of variability for at least one key outcome no $\square$ yes $\square$ where:

no $\square$ yes $\square$ where:

no $\square$ yes $\square$ where:

no $\square$ yes $\square$ where:

no $\square$ yes $\square$ where:

no $\square$ yes $\square$ where:

no $\square$ yes $\square$ where:

no $\square$ yes $\square$ where:

no $\square$ yes $\square$ where:

no $\square$ yes $\square$ where:

no $\square$ yes $\square$ where:

Figure 2 The Physiotherapy Evidence Database (PEDro) scale; from Maher et al. ${ }^{49}$

continuous outcomes will be analysed using mean difference or standardised mean difference, both with their 95\% CIs. Meta-analysis will be done separately on trials that evaluated the effects of MCEs in patients after surgery and on trials in which patients with symptomatic LDH had not undergone surgery. All data from the meta-analyses with $95 \%$ CI will be reported in forest plots.

\section{Analysis problems}

If sufficient homogeneous studies are available for statistical pooling, we will conduct a meta-analysis for the time points: short $(<3$ months after the baseline measurements were taken), intermediate (at least 3 months but $<12$ months after the baseline measurements were taken) and long-term (12 months or more after the baseline measurements were taken) follow-up. If multiple time points fall within the same category, we will use the one that is closest to the end of the treatment, 6 and 12 months. ${ }^{27}$

\section{Subgroup analysis}

Subgroup analysis will be conducted for the population. The results will be split into two separate analyses: one population with presurgery symptomatic LDH (or a population who had not undergone surgery) and the other with postsurgery symptomatic LDH. Furthermore, subgroup analysis will be performed for the secondary outcomes (functional tests, muscle thickness, quality of life, return to work, muscle endurance and adverse events).

\section{Sensitivity analysis}

A sensitivity analysis will be performed to test the impact of the results on the RoB assessment rated by the PEDro scale. ${ }^{49}$ We will also implement sensitivity analyses to explore the effects of methodological quality and sample size on the robustness of review conclusions. Sensitivity analyses will be reported with a summary table.

\section{Summary of findings table}

We will evaluate the quality of evidence for the main comparison at the outcome level using the Grading of Recommendations Assessment, Development and Evaluation (GRADE) approach. ${ }^{58} \mathrm{~A}$ 'summary of findings' table will be produced in our systematic review. Five domains will be judged for each outcome in the main comparison: limitations in study design and execution (RoB), inconsistency of results, indirectness, imprecision and publication bias (reporting bias). In this systematic review and meta-analysis, limitations in the study design and execution will be assessed using the PEDro scale. ${ }^{49}$ If studies have major limitations, our confidence in the estimate of the treatment effect decreases. Therefore, the quality of evidence will be downgraded. ${ }^{58}$ We will consider four criteria to assess inconsistency in results: point estimates, CIs, the statistical test for heterogeneity 
and the $\mathrm{I}^{2}$ test. ${ }^{59}$ Indirectness will be judged by looking at the evidence tables for the target population, intervention, comparison or outcome. ${ }^{58}$ In addition, results will be considered to be imprecise when studies include relatively few patients and few events but have a wide CI around the estimate of the effect. ${ }^{58}$ Finally, when a sufficient number of studies $(\geq 10)$ are identified, publication bias will be assessed by visually examining funnel plots for evidence of asymmetry. The overall quality of the evidence supporting each outcome will be graded as high, moderate, low or very low.

- High-quality evidence will be identified when there are consistent findings among at least $75 \%$ of included trials. ${ }^{27} 43{ }^{60}$ No study design limitation is found and the data are consistent, direct and precise with no publication bias. In addition, further research is unlikely to change confidence in the estimate of effect.

- Moderate-quality evidence will be identified when one of the five domains is not met. ${ }^{27} 4{ }^{60}$ In this level, we are moderately confident about the effect estimate. However, further research is likely to affect confidence in the estimated effect and may change that estimate.

- Low-quality evidence will be identified when two of the five domains are not met. ${ }^{27} 43{ }^{60}$ In this level, our confidence in the effect estimate is limited. Further research is likely to have an important impact on confidence in the estimated effect and is likely to change that estimate.

- Very low-quality evidence will be identified when three of the five domains are not met. ${ }^{27} 43{ }^{60}$ In this level, we have little confidence in the effect estimate. Any estimate of the effect is uncertain.

- No evidence will be identified when none of the five domains are met.

Online supplementary appendix 2 provides more details about the five domains and the quality of evidence.

\section{Ethics and dissemination}

Ethics approval is not required because this is a protocol for a systematic review and patients will not be involved in this research. The results of this study will be submitted to a peer-reviewed journal for publication and will also be presented at national and international academic and clinical conferences.

\section{DISCUSSION}

LDH is the most common cause of activity limitation in people aged $<45$ years. ${ }^{61}$ Furthermore, this condition is among the most common causes of LBP or sciatica (radicular leg pain) and imposes a heavy burden on both the individual and society. ${ }^{62-64}$ Different kinds of non-invasive and invasive (surgical) treatment strategies have been suggested, with varying degrees of success. ${ }^{63}$ Treatment often includes patient education, physical therapy, alternative medicine options and pharmacotherapy. If these strategies fail, surgical intervention is usually recommended. Physical therapy is considered important for the conservative management of this problem. ${ }^{16}$ Therapeutic exercises are physical therapy interventions that effectively alleviate pain and improve function for patients with LDH. ${ }^{10}$ MCEs-one type of active therapeutic exercises-are a popular approach for clinicians and researchers in the conservative managements of patients with LDH. To date, several systematic reviews have been published on this topic. ${ }^{6}{ }^{34}$ However, existing systematic reviews are out of date and none of them compared MCEs with other conservative physiotherapy interventions.

As stated earlier, non-invasive conservative treatment is the first-line choice for most patients with $\mathrm{LDH} .{ }^{16}$ Hence, a systematic review and meta-analysis is needed to evaluate the effectiveness of MCEs for symptomatic LDH. The databases which will be searched in our systematic review, the primary and secondary outcomes and the method of meta-analysis are clarified in this protocol. Our systematic review with meta-analysis will help clinicians, therapists and patients to gain a better understanding of the effectiveness of MCEs. We will try to include grey literature since some high-quality studies have not yet been published. In addition, no limitation by language will be imposed. Therefore, the limitations of this systematic review will be reduced and conclusions about the results will be improved. In this review, meta-analysis will be conducted on trials in which patients with LDH had undergone surgery and also on trials in which the patients had not undergone surgery. Our results will be important to clarify which type of exercise is most effective in LDH. Implications for future research can be drawn from the results.

\section{Author affiliations}

${ }^{1}$ Department of Physiotherapy, School of Rehabilitation Sciences, Iran University of Medical Sciences and Health Services, Tehran, Iran

${ }^{2}$ Student Research Committee, University of Social Welfare and Rehabilitation Sciences, Tehran, Iran

${ }^{3}$ Department of Rehabilitation Basic Sciences, School of Rehabilitation Sciences, Iran University of Medical Sciences and Health Services, Tehran, Iran

${ }^{4}$ Pediatric Neurorehabilitation Research Center, University of Social Welfare and Rehabilitation Sciences, Tehran, Iran

${ }^{5}$ Faculty of Allied Health Sciences, University Institute of Physical Therapy, University of Lahore, Lahore, Pakistan

${ }^{6}$ Department of Health Sciences Education Development, School of Public Health, Tehran University of Medical Sciences and Health Services, Tehran, Iran

Acknowledgements The authors acknowledge the efforts of AAK for his help in developing this protocol. We also particularly thank the reviewers for their valuable comments, which helped considerably to improve the quality of the manuscript.

Contributors MRP, MT, IET, MAS, MAM-B and AAK conceived and designed the study. MRP, MT, MAS and AAK developed the search strategies and contributed to analysis of the studies. MRP and MT were responsible for the initial drafting, edited the manuscript and approved the manuscript for submission. IET, MAM-B and AAK revised the manuscript. MRP and MT will also screen potential studies, extract data and assess their quality. Any 
discrepancies will be resolved by consensus between MRP and MT. When consensus is not reached, MAS will act as arbitrator.

Competing interests None declared.

Provenance and peer review Not commissioned; externally peer reviewed.

Data sharing statement All recorded information from the data extraction process, not included in the systematic review article, will be available on request.

Open Access This is an Open Access article distributed in accordance with the Creative Commons Attribution Non Commercial (CC BY-NC 4.0) license, which permits others to distribute, remix, adapt, build upon this work noncommercially, and license their derivative works on different terms, provided the original work is properly cited and the use is non-commercial. See: http:// creativecommons.org/licenses/by-nc/4.0/

\section{REFERENCES}

1. Yang $\mathrm{H}$, Liu $\mathrm{H}$, Li Z, et al. Low back pain associated with lumbar disc herniation: role of moderately degenerative disc and annulus fibrous tears. Int J Clin Exp Med 2015;8:1634-44.

2. Schoenfeld AJ, Weiner BK. Treatment of lumbar disc herniation: evidence-based practice. Int J Gen Med 2010;3:209-14.

3. Anderson PA, McCormick PC, Angevine PD. Randomized controlled trials of the treatment of lumbar disk herniation: 1983-2007. J Am Acad Orthop Surg 2008;16:566-73.

4. Jordan J, Konstantinou K, O'Dowd J. Herniated lumbar disc. BMJ Clin Evid 2009:3:1-34.

5. Ozkanli S, Kaner T, Efendioglu M, et al. The relation of matrix metalloproteinase 1, 2, 3 expressions with clinical and radiological findings in primary and recurrent lumbar disc herniations. Turk Neurosurg 2015;25:111-16.

6. Hahne AJ, Ford JJ, McMeeken JM. Conservative management of lumbar disc herniation with associated radiculopathy: a systematic review. Spine (Phila Pa 1976) 2010;35:E488-504.

7. Koes BW, van Tulder MW, Peul WC. Diagnosis and treatment of sciatica. BMJ 2007;334:1313-17.

8. Boden SD, Davis DO, Dina TS, et al. Abnormal magnetic-resonance scans of the lumbar spine in asymptomatic subjects. A prospective investigation. J Bone Joint Surg Am 1990;72:403-8.

9. Jensen MC, Brant-Zawadzki MN, Obuchowski N, et al. Magnetic resonance imaging of the lumbar spine in people without back pain. N Engl J Med 1994;331:69-73

10. Ahmed S, Hassan T, Hanif A. Effects of lumbar stabilization exercise in management of pain and restoration of function in patients with postero lateral disc herniation. Ann King Edward Med Univ 2012;18.

11. Gregory DS, Seto CK, Wortley GC, et al. Acute lumbar disk pain: navigating evaluation and treatment choices. Am Fam Physician 2008;78:835-42.

12. Zhang YG, Sun Z, Liu J, et al. Advances in susceptibility genetics of intervertebral degenerative disc disease. Int J Biol Sci 2008;4:283-90.

13. Rajagopal TS, Marshall RW. Chapter 28: Microdiscectomy. In: Bentley G, ed. European surgical orthopaedics and traumatology: the EFORT textbook. Springer Berlin Heidelberg 2014:557-80.

14. Paoloni M, Di Sante L, Cacchio A, et al. Intramuscular oxygen-ozone therapy in the treatment of acute back pain with lumbar disc herniation: a multicenter, randomized, double-blind, clinical trial of active and simulated lumbar paravertebral injection. Spine (Phila Pa 1976) 2009;34:1337-44.

15. Pearson AM, Blood EA, Frymoyer JW, et al. SPORT lumbar intervertebral disk herniation and back pain: does treatment, location, or morphology matter? Spine (Phila Pa 1976) 2008;33:428.

16. Eckel TS. Advances in spinal imaging and interventions (abstr). 40th annual meeting of the American Society of Neuroradiology, Vancouver, 2002

17. Silby $\mathrm{H}$. Conservative management of lumbar disk herniation. Postgrad Med 1988;84:157-72.

18. Brötz D, Küker W, Maschke E, et al. A prospective trial of mechanical physiotherapy for lumbar disk prolapse. J Neurol 2003;250:746-9.

19. Pinheiro-Franco JL, Vaccaro AR, Benzel EC, et al. Advanced concepts in lumbar degenerative disk disease. Springer, 2015.

20. Kim Y-S, Park J, Shim JK. Effects of aquatic backward locomotion exercise and progressive resistance exercise on lumbar extension strength in patients who have undergone lumbar diskectomy. Arch Phys Med Rehabil 2010;91:208-14.

21. Akuthota V, Ferreiro A, Moore T, et al. Core stability exercise principles. Curr Sports Med Rep 2008;7:39-44.
22. Shamsi MB, Sarrafzadeh J, Jamshidi A. Comparing core stability and traditional trunk exercise on chronic low back pain patients using three functional lumbopelvic stability tests. Physiother Theory Pract 2015;31:89-98.

23. Costa LO, Maher CG, Latimer J, et al. Motor control exercise for chronic low back pain: a randomized placebo-controlled trial. Phys Ther 2009;89:1275-86.

24. Hodges PW, Richardson CA. Inefficient muscular stabilization of the lumbar spine associated with low back pain: a motor control evaluation of transversus abdominis. Spine (Phila Pa 1976) 1996;21:2640-50.

25. Akuthota V, Nadler SF. Core strengthening. Arch Phys Med Rehabil 2004;85:S86-92.

26. Richardson CA, Hodges $P$, Hides J. Therapeutic exercise for lumbopelvic stabilization: a motor control approach for the treatment and prevention of low back pain. Churchill Livingstone, 2004.

27. Saragiotto BT, Maher CG, Yamato TP, et al. Motor control exercise for chronic non-specific low-back pain. The Cochrane Library, 2016.

28. Comerford M, Mottram S. Kinetic control: the management of uncontrolled movement. Australia: Elsevier, 2012.

29. Hodges PW, Richardson CA. Delayed postural contraction of transversus abdominis in low back pain associated with movement of the lower limb. J Spinal Disord 1998;11:46-56.

30. Hodges PW, Richardson CA. Altered trunk muscle recruitment in people with low back pain with upper limb movement at different speeds. Arch Phys Med Rehabil 1999;80:1005-12.

31. Fortin M, Lazáry A, Varga PP, et al. Paraspinal muscle asymmetry and fat infiltration in patients with symptomatic disc herniation. Eur Spine J 2016;25:1452-9.

32. Franke J, Hesse T, Tournier C, et al. Morphological changes of the multifidus muscle in patients with symptomatic lumbar disc herniation: clinical article. J Neurosurg Spine 2009;11:710-14.

33. Newcomer K, Laskowski ER, Yu B, et al. Repositioning error in low back pain. Comparing trunk repositioning error in subjects with chronic low back pain and control subjects. Spine (Phila Pa 1976) 2000;25:245-50.

34. Jacobs WC, van Tulder M, Arts $M$, et al. Surgery versus conservative management of sciatica due to a lumbar herniated disc: a systematic review. Eur Spine J 2011;20:513-22.

35. Shin B-J. Risk factors for recurrent lumbar disc herniations. Asian Spine J 2014:8:211-15.

36. Bae SH, Lee HG, Kim YE, et al. Effects of trunk stabilization exercises on different support surfaces on the cross-sectional area of the trunk muscles and balance ability. J Phys Ther Sci 2013;25:741-5.

37. Kennedy DJ, Noh MY. The role of core stabilization in lumbosacral radiculopathy. Phys Med Rehabil Clin N Am 2011;22:91-103.

38. Ostelo RW, Costa LOP, Maher CG, et al. Rehabilitation after lumbar disc surgery: an update Cochrane review. Spine (Phila Pa 1976) 2009;34:1839-48.

39. Liberati A, Altman DG, Tetzlaff J, et al. The PRISMA statement for reporting systematic reviews and meta-analyses of studies that evaluate health care interventions: explanation and elaboration. Ann Intern Med 2009;151:W-65-94.

40. Fagerlund MK, Thelander U. Comparison of myelography and computed tomography in establishing lumbar disc herniation. Acta Radiol 1989;30:241-6.

41. Fardon DF, Milette PC. Nomenclature and classification of lumbar disc pathology: recommendations of the combined task forces of the North American Spine Society, American Society of Spine Radiology and American Society of Neuroradiology. Spine (Phila Pa 1976) 2001;26:E93-113.

42. O'Sullivan PB, Phyty GDM, Twomey LT, et al. Evaluation of specific stabilizing exercise in the treatment of chronic low back pain with radiologic diagnosis of spondylolysis or spondylolisthesis. Spine (Phila Pa 1976) 1997;22:2959-67.

43. Macedo LG, Saragiotto BT, Yamato TP, et al. Motor control exercise for acute non-specific low back pain. The Cochrane Library, 2016.

44. Herrington L, Davies R. The influence of Pilates training on the ability to contract the transversus abdominis muscle in asymptomatic individuals. J Bodywork Mov Ther 2005;9:52-7.

45. Godin K, Stapleton J, Kirkpatrick SI, et al. Applying systematic review search methods to the grey literature: a case study examining guidelines for school-based breakfast programs in Canada. Syst Rev 2015;4:138.

46. Higgins JP, Green S. Cochrane handbook for systematic reviews of interventions. Wiley Online Library, 2008.

47. Eden J, Levit L, Berg A, et al. Finding what works in health care: standards for systematic reviews. National Academies Press, 2011

48. Dillman DA. Mail and Internet surveys: The tailored design method2007 Update with new Internet, visual and mixed-mode guide. John Wiley \& Sons, 2011. 
49. Maher CG, Sherrington C, Herbert RD, et al. Reliability of the PEDro scale for rating quality of randomized controlled trials. Phys Ther 2003;83:713-21.

50. Verhagen AP, de Vet $\mathrm{HC}$, de Bie RA, et al. The Delphi list: a criteria list for quality assessment of randomized clinical trials for conducting systematic reviews developed by Delphi consensus. J Clin Epidemiol 1998;51:1235-41.

51. Sherrington C, Herbert RD, Maher CG, et al. PEDro. A database of randomized trials and systematic reviews in physiotherapy. Man Ther 2000;5:223-6.

52. Smith BE, Littlewood C, May S. An update of stabilisation exercises for low back pain: a systematic review with meta-analysis. BMC Musculoskelet Disord 2014:15:416.

53. May S, Johnson R. Stabilisation exercises for low back pain: a systematic review. Physiotherapy 2008;94:179-89.

54. Wang XQ, Zheng JJ, Yu ZW, et al. A meta-analysis of core stability exercise versus general exercise for chronic low back pain. PLOS ONE 2012;7:e52082.

55. Deeks J, Higgins J, Altman D. Chapter 9: analysing data and undertaking meta-analysis. In: Higgins JPT, Green S, eds. Cochrane handbook for systematic reviews. Wiley-Blackwell, 2011:243-96.

56. Borenstein M, Hedges LV, Higgins J, et al. A basic introduction to fixed-effect and random-effects models for meta-analysis. Res Synth Methods 2010;1:97-111.
57. Grimes CA, Bolhuis DP, He FJ, et al. Dietary sodium intake and overweight and obesity in children and adults: a protocol for a systematic review and meta-analysis. Syst Rev 2016;5:7.

58. Schünemann $\mathrm{H}$, Brozek J, Oxman $\mathrm{A}$. GRADE handbook for grading quality of evidence and strength of recommendation. The GRADE Working Group, 2009.

59. Guyatt GH, Oxman AD, Kunz R, et al. GRADE guidelines: 7. Rating the quality of evidence-inconsistency. J Clin Epidemiol 2011;64:1294-302.

60. Yamato TP, Maher CG, Saragiotto BT, et al. Pilates for low back pain: complete republication of a cochrane review. Spine (Phila Pa 1976) 2016;41:1013-21.

61. Andersson GB. Epidemiological features of chronic low-back pain. Lancet 1999;354:581-5.

62. Atlas SJ, Keller RB, Wu YA, et al. Long-term outcomes of surgical and nonsurgical management of sciatica secondary to a lumbar disc herniation: 10 year results from the maine lumbar spine study. Spine (Phila Pa 1976) 2005;30:927-35.

63. Awad JN, Moskovich R. Lumbar disc herniations: surgica versus nonsurgical treatment. Clin Orthop Relat Res 2006;443:183-97.

64. Lee J, Shin JS, Lee YJ, et al. Effects of Shinbaro pharmacopuncture in sciatic pain patients with lumbar disc herniation: study protocol for a randomized controlled trial. Trials 2015;16:455. 